\title{
AN OPERATIONAL HAAR WAVELET METHOD FOR SOLVING FRACTIONAL VOLTERRA INTEGRAL EQUATIONS
}

\author{
HABIBOLlah SAEEDI $*, * *$, NASIBEH MOLLAHASANI $*, * *$, \\ MAHMOUD MOHSENI MOGHADAM ${ }^{* * *}$, GENNADY N. CHUEV ${ }^{* *, * * * *}$ \\ * Department of Mathematics, Faculty of Mathematics and Computer Science \\ Shahid Bahonar University of Kerman, Kerman, 76169-14111, Iran \\ e-mail: habibsaeedi@gmail.com \\ ** Max Planck Institute for Mathematics in the Sciences (MIS) \\ Inselstrasse 22, Leipzig 04103, Germany \\ *** Mahani Mathematical Research Center \\ Shahid Bahonar University of Kerman, Kerman, 76169-14111, Iran \\ **** Institute of Theoretical and Experimental Biophysics \\ Russian Academy of Sciences, Pushchino, Moscow Region 142290, Russia
}

\begin{abstract}
A Haar wavelet operational matrix is applied to fractional integration, which has not been undertaken before. The Haar wavelet approximating method is used to reduce the fractional Volterra and Abel integral equations to a system of algebraic equations. A global error bound is estimated and some numerical examples with smooth, nonsmooth, and singular solutions are considered to demonstrate the validity and applicability of the developed method.
\end{abstract}

Keywords: fractional Volterra integral equation, Abel integral equation, fractional calculus, Haar wavelet method, operational matrices.

\section{Introduction}

The development of the theory of fractional integrals and derivatives starts with Euler, Liouville and Abel (1823). However, during the last ten years, fractional calculus has attracted much more attention of physicists and mathematicians. In fact, real problems in scientific fields such as physics, mechanics, chemistry and biology are formulated as partial differential equations or integral equations. Many authors have demonstrated applications of fractional calculus to coelastic materials (Bagley and Torvik, 1985), continuum and statistical mechanics (Mainardi, 1997), colored noise (Mandelbrot, 1967), economics (Baillie, 1996), bioengineering (Magin, 2004), anomalous diffusion and transport (Chena et al., 2010), the dynamics of interfaces between nanoparticles and substrates (Chow, 2005), complex viscoelasticity (Meral et al., 2010), rheology (Metzler, 2003) and others. There are also several methods for solving fractional integral equations like He's homotopy (Pandey et al., 2009), Adomian decomposition ( $\mathrm{Li}$ and Wang, 2009), collocation method (Lepik, 2009) and power spectral density (Zaman and Yu, 1995).

The aim of this paper is to introduce a new operational wavelet method for approximating the solution of a fractional Volterra integral equation in the following form:

$$
\begin{array}{r}
f(x)-\frac{1}{\Gamma(\alpha)} \int_{0}^{x}(x-t)^{\alpha-1} k(x, t) f(t) \mathrm{d} t=g(x), \\
0 \leq x \leq 1 .
\end{array}
$$

The kernel $k(x, t)$ and the right-hand-side function $g(x)$ are given, and $\alpha>0$ is a real number. This equation is also referred to as the weakly-singular linear Volterra integral equation (see Hachbusch, 1995). The value $\alpha=1$ corresponds to the ordinary (non-fractional) Volterra integral equation. Particularly, if $k(x, t)=1$ and $0<\alpha<1$ in Eqn. (1), we have an Abel integral equation in the form

$$
f(x)-\lambda \int_{0}^{x} \frac{f(t)}{(x-t)^{\beta}} \mathrm{d} t=g(x), \quad 0<\beta<1,
$$


where $\lambda=1 / \Gamma(\alpha)$ and $\beta=1-\alpha$.

The treatment of Eqn. (1) is not simple because, as is well known, the solutions of weakly singular Volterra integral equations usually have a weak singularity at $x=$ 0 , even when the inhomogeneous term $g(x)$ is smooth. Miller and Feldstein (1971) proved that the solution of (1) is unique and continuous in $[0,1]$ if $g \in L^{1}(0,1)$ and $k \in L^{\infty}$. A deeper insight into this problem is provided by many differentiability results for $f(x)$ obtained by various authors under specific hypotheses on $g(x)$ and $k(x, t)$ (Miller and Feldstein, 1971).

A possibility of nonsmooth solution complicates the numerical investigation of Eqn. (2). Various numerical techniques have been developed to treat such nonsmooth solutions (Baratella and Orsi, 2004; Brunner, 1984; Dixon, 1985). In general, the numerical solution of Eqn. (1) is often quite complicated, so we are looking for simplifications. For this reason we use an operational Haar wavelet method, since Haar wavelets are the simpest ones (Lepik, 2003; 2004, Maleknejad, 2005; Hsiao, 2007), and it has not received much attention so far. Only Lepik (2009) has used Haar wavelets to solve such equations. He has utilized these wavelets as a collocation method and introduced a local error estimate, but we use Haar wavelets to obtain an operational method which yields an operational matrix of fractional integration.

The main characteristic of an operational method is to convert a differential equation into an algebraic one. This not only simplifies the problem but also speeds up the computation. However, the interest in the wavelet treatment of various integral equations has recently increased due to promising applications of this method in computational chemistry (Chuev and Fedorov, 2004a; 2004b; 2004c; Fedorov, 2004; Fedorov and Khoromskij, 2007; Fedorov and Chuev, 2005).

In the present paper we first introduce Haar wavelets and their properties, then construct a new operational matrix of fractional integration via Haar wavelets. After that the method is described and its convergence is discussed. A global error estimate is also evaluated and several representative examples are considered. We should remark that, despite numerous examples of numerical evaluations of the fractional Volterra integral equation, a general theory guaranteeing the convergence of the solution for this equation is still incomplete (Baratella and Orsi, 2004; Brunner, 1984; Dixon, 1985; Miller and Feldstein, 1971). Therefore, to consider the conditions under which the method will fail, we shall apply our approach not only to smooth solutions, but also to those revealing nonsmooth behavior.

\section{Function approximation}

The orthogonal set of the Haar wavelets $h_{n}(x)$ is a group of square waves defined as follows:

$$
\begin{gathered}
h_{0}(x)= \begin{cases}1, & 0 \leq x<1, \\
0, & \text { elsewhere, }\end{cases} \\
h_{1}(x)= \begin{cases}1, & 0 \leq x<\frac{1}{2}, \\
-1, & \frac{1}{2} \leq x<1, \\
0, & \text { elsewhere, }\end{cases} \\
h_{n}(x)=h_{1}\left(2^{j} x-k\right), \\
n=2^{j}+k, \quad j, k \in \mathbb{N} \cup\{0\}, \quad 0 \leq k<2^{j},
\end{gathered}
$$

such that

$$
\int_{0}^{1} h_{n}(x) h_{m}(x) \mathrm{d} x=2^{-j} \delta_{n m},
$$

where $\delta_{n m}$ is the Kronecker delta. For more details, see the works of Akansu and Haddad (1981), Vetterli and Kovacevic (1995), or Strang (1989).

The Heaviside step function is defined as

$$
u(x)= \begin{cases}1, & x \geq 0, \\ 0, & x<0 .\end{cases}
$$

A useful property of this function is

$$
u(x-a) u(x-b)=u(x-\max \{a, b\}), \quad a, b \in \mathbb{R} .
$$

Note that we can write Eqn. (3) by using the Heaviside step function as

$$
\begin{aligned}
h_{0}(x)= & u(x)-u(x-1), \\
h_{n}(x)= & u\left(x-\frac{k}{2^{j}}\right)-2 u\left(x-\frac{k+1 / 2}{2^{j}}\right) \\
& +u\left(x-\frac{k+1}{2^{j}}\right), \\
n= & 2^{j}+k, \quad j, k \in \mathbb{N} \cup\{0\}, \quad 0 \leq k<2^{j} .
\end{aligned}
$$

Each square integrable function $f(x)$ in the interval $[0,1)$ can be expanded into a Haar series of infinite terms:

$$
\begin{aligned}
f(x)= & c_{0} h_{0}(x) \\
& +\sum_{j=0}^{\infty} \sum_{k=0}^{2^{j}-1} c_{2^{j}+k} h_{2^{j}+k}(x), \quad x \in[0,1],
\end{aligned}
$$

where the Haar coefficients are determined as

$$
\begin{aligned}
c_{i} & =2^{j} \int_{0}^{1} f(x) h_{i}(x) \mathrm{d} x, \\
i & =0,2^{j}+k, \quad j, k \in \mathbb{N} \cup\{0\}, \quad 0 \leq k<2^{j},
\end{aligned}
$$


such that the following integral square error $\epsilon_{m}$ is minimized:

$$
\begin{aligned}
\epsilon_{m}=\int_{0}^{1}\left[f(x)-\sum_{i=0}^{m-1} c_{i} h_{i}(x)\right]^{2} \mathrm{~d} x, & \\
& m=2^{J+1}, \quad J \in \mathbb{N} \cup\{0\} .
\end{aligned}
$$

By using Eqn. (5), the above Haar coefficients can be rewritten as

$$
\begin{gathered}
c_{i}=2^{j}\left[\int_{\frac{k}{2^{j}}}^{\frac{k+1 / 2}{2^{j}}} f(x) \mathrm{d} x-\int_{\frac{k+1 / 2}{2^{j}}}^{\frac{k+1}{2^{j}}} f(x) \mathrm{d} x\right], \\
i=2^{j}+k, \quad j, k \in \mathbb{N} \cup\{0\}, \quad 0 \leq k<2^{j} .
\end{gathered}
$$

If $f(x)$ is piecewise constant or may be approximated by a piecewise constant function during each subinterval, the series sum in Eqn. (6) can be truncated after $m$ terms ( $m=2^{J+1}, J \geq 0$ being the resolution level of the wavelet), that is

$$
\begin{aligned}
f(x) & \cong c_{0} h_{0}(x)+\sum_{j=0}^{J} \sum_{k=0}^{2^{j}-1} c_{2^{j}+k} h_{2^{j}+k}(x) \\
& =\mathbf{c}^{T} \mathbf{h}(x)=f_{m}(x), \quad x \in[0,1],
\end{aligned}
$$

where $\mathbf{c}=\mathbf{c}_{m \times 1}=\left[\begin{array}{cc}c_{0}, & c_{1}, \ldots, c_{m-1}\end{array}\right]^{T}, \mathbf{h}(x)=$ $\mathbf{h}_{m \times 1}(x)=\left[h_{0}(x), h_{1}(x), \ldots, h_{m-1}(x)\right]^{T}$.

\section{Haar wavelet operational matrix of fractional integration}

In this section we obtain an operational matrix of fractional integration, based on Haar wavelets, which is novel. The Riemann-Liouville fractional integral operator of order $\alpha>0$ of the function $f(x)$ is defined as (Podlubny, 1999; Miller and Ross, 1993)

$$
\begin{aligned}
I^{\alpha} f(x)=\frac{1}{\Gamma(\alpha)} \int_{0}^{x}(x-t)^{\alpha-1} f(t) \mathrm{d} t, & \\
\alpha>0, & x>0,
\end{aligned}
$$

where $\Gamma(\cdot)$ is the Gamma function with the property $\Gamma(x+$ $1)=x \Gamma(x), x \in \mathbb{R}$. Some properties of the operator $I^{\alpha}$ can be found in the research by Podlubny (1999). We mention only the following: For $\alpha \geqslant 0$ and $\gamma>-1$, we have

$$
I^{\alpha} x^{\gamma}=\frac{\Gamma(\gamma+1)}{\Gamma(\alpha+\gamma+1)} x^{\alpha+\gamma} .
$$

The integration of $\mathbf{h}(x)$ can be expanded into a Haar series with a Haar coefficient matrix $\mathbf{P}_{m}$ (Chen and Hsiao, 1997) as

$$
\int_{0}^{x} \mathbf{h}(x) \mathrm{d} x \cong \mathbf{P}_{m} \mathbf{h}(x)
$$

The $m \times m$ matrix $\mathbf{P}_{m}$ is called the operational matrix of integration and is given by Hsiao and Wu (2007) as

$$
\mathbf{P}_{m}=\frac{1}{2 m}\left[\begin{array}{cc}
2 m \mathbf{P}_{m / 2} & -\mathbf{H}_{m / 2 \times m / 2} \\
\mathbf{H}_{m / 2 \times m / 2}^{-1} & \mathbf{0}
\end{array}\right],
$$

where $\mathbf{H}_{1 \times 1}=[1], \mathbf{P}_{1}=[1 / 2]$ and

$$
\mathbf{H}_{m \times m}=\left[\mathbf{h}\left(\frac{1}{2 m}\right), \mathbf{h}\left(\frac{3}{2 m}\right), \ldots, \mathbf{h}\left(\frac{2 m-1}{2 m}\right)\right] .
$$

Three basic multiplication properties of Haar wavelets are as follows (Hsiao and Wu, 2007):

(i) $h_{n}(x) h_{0}(x)=h_{n}(x)$ for any $n \in \mathbb{N} \cup\{0\}$.

(ii) For any two Haar wavelets $h_{n}(x)$ and $h_{l}(x)$ with $n<$ $l$, we have

$$
h_{n}(x) h_{l}(x)=\rho_{n l} h_{l}(x)
$$

where

$$
\begin{aligned}
& \rho_{n l} \\
& \quad=h_{n}\left(2^{-i}(q+1 / 2)\right) \\
& \quad= \begin{cases}1, & 2^{i-j} k \leq q<2^{i-j}(k+1 / 2), \\
-1, & 2^{i-j}(k+1 / 2) \leq q<2^{i-j}(k+1), \\
0, & \text { elsewhere, }\end{cases}
\end{aligned}
$$

for $n=2^{j}+k, \quad j \geq 0, \quad 0 \leq k<2^{j}$ and $l=$ $2^{i}+q, \quad i \geq 0, \quad 0 \leq q<2^{i}$

(iii) The square of any Haar wavelet is a block pulse with a magnitude of 1 during both positive and negative half waves.

The product of $\mathbf{h}(x), \mathbf{h}^{T}(x)$ and $\mathbf{c}$ can also be expanded into a Haar series with a Haar coefficient matrix $\mathbf{M}_{m}$ as follows:

$$
\mathbf{h}(x) \mathbf{h}^{T}(x) \mathbf{c}=\mathbf{M}_{m} \mathbf{h}(x),
$$

where $\mathbf{M}_{m}$ is an $m \times m$ matrix referred to as the product operational matrix and given by Hsiao and Wu (2007):

$$
\mathbf{M}_{m}=\left[\begin{array}{cc}
\mathbf{M}_{m / 2} & \mathbf{H}_{m / 2} \operatorname{diag}\left(\widetilde{\mathbf{c}}_{b}\right) \\
\operatorname{diag}\left(\widetilde{\mathbf{c}}_{b}\right) \mathbf{H}_{m / 2}^{-1} & \operatorname{diag}\left(\widetilde{\mathbf{c}}_{a}^{T} \mathbf{H}_{m / 2}\right)
\end{array}\right],
$$

such that $\mathbf{M}_{1}=c_{0}$ and

$$
\widetilde{\mathbf{c}}_{a}=\left[c_{0}, \ldots, c_{m / 2-1}\right]^{T}, \quad \widetilde{\mathbf{c}}_{b}=\left[c_{m / 2}, \ldots, c_{m-1}\right]^{T} .
$$

Now we want to obtain the operational matrix of fractional integration for Haar wavelets, which is a generalized form of $\mathbf{P}_{m}$ in (12). The fractional integration of 
order $\alpha$ of $\mathbf{h}(x)$ can be expanded into a Haar series with a Haar coefficient matrix $\mathbf{P}_{m}^{\alpha}$ as follows:

$$
\frac{1}{\Gamma(\alpha)} \int_{0}^{x}(x-t)^{\alpha-1} \mathbf{h}(t) \mathrm{d} t=\mathbf{P}_{m}^{\alpha} \mathbf{h}(x) .
$$

We call this $m \times m$ square matrix $\mathbf{P}_{m}^{\alpha}$ the (generalized) operational matrix of fractional integration. Thus for expanding the Riemann-Liouville integral, it is enough to expand

$$
\frac{1}{\Gamma(\alpha)} \int_{0}^{x}(x-t)^{\alpha-1} h_{n}(t) \mathrm{d} t
$$

for $n=0,1, \ldots, m-1$, in a Haar series. We know that

$$
\frac{1}{\Gamma(\alpha)} \int_{0}^{x}(x-t)^{\alpha-1} h_{n}(t) \mathrm{d} t=\frac{1}{\Gamma(\alpha)}\left\{x^{\alpha-1} * h_{n}(x)\right\},
$$

where $*$ is the convolution operator of two functions. By taking the Laplace transform of the above equation, we have

$$
\begin{aligned}
\mathcal{L}\left\{\frac{1}{\Gamma(\alpha)} \int_{0}^{x}(x-t)^{\alpha-1} h_{n}(t) \mathrm{d} t\right\} \\
=\frac{1}{\Gamma(\alpha)} \mathcal{L}\left\{x^{\alpha-1}\right\} \mathcal{L}\left\{h_{n}(x)\right\}
\end{aligned}
$$

where

$$
\begin{aligned}
\mathcal{L}\left\{x^{\alpha-1}\right\}= & \frac{\Gamma(\alpha)}{s^{\alpha}}, \\
\mathcal{L}\left\{h_{n}(x)\right\}= & \mathcal{L}\left\{u\left(x-\frac{k}{2^{j}}\right)-2 u\left(x-\frac{k+1 / 2}{2^{j}}\right)\right. \\
& \left.+u\left(x-\frac{k+1}{2^{j}}\right)\right\} \\
= & \frac{1}{s}\left\{e^{-\frac{k}{2^{j}} s}-2 e^{-\frac{k+1 / 2}{2^{j}} s}+e^{-\frac{k+1}{2^{j}} s}\right\} .
\end{aligned}
$$

The last two equalities are obtained using the properties of the Laplace transform. Therefore, (16) can be rewritten as

$$
\begin{aligned}
\mathcal{L}\left\{\frac{1}{\Gamma(\alpha)} \int_{0}^{x}(x-t)^{\alpha-1} h_{n}(t) \mathrm{d} t\right\} \\
=\frac{1}{\Gamma(\alpha+1)} \cdot \frac{\Gamma(\alpha+1)}{s^{\alpha+1}} \\
\cdot\left\{e^{-\frac{k}{2^{j}} s}-2 e^{-\frac{k+1 / 2}{2^{j}} s}+e^{-\frac{k+1}{2^{j}} s}\right\} .
\end{aligned}
$$

Now, taking the inverse Laplace transform of the above equation, we find

$$
\begin{aligned}
& \frac{1}{\Gamma(\alpha)} \int_{0}^{x}(x-t)^{\alpha-1} h_{n}(t) \mathrm{d} t \\
& =\frac{1}{\Gamma(\alpha+1)}\{\underbrace{\left(x-\frac{k}{2^{j}}\right)^{\alpha} u\left(x-\frac{k}{2^{j}}\right)}_{X(x)} \\
& -\underbrace{}_{Y(x)} \underbrace{\left(x-\frac{k+1 / 2}{2^{j}}\right)^{\alpha} u\left(x-\frac{k+1 / 2}{2^{j}}\right)}_{Z(x)}
\end{aligned}
$$

$$
=\frac{1}{\Gamma(\alpha+1)}\{X(x)-2 Y(x)+Z(x)\} .
$$

Specifically, for $n=0$, we have

$$
\frac{1}{\Gamma(\alpha)} \int_{0}^{x}(x-t)^{\alpha-1} h_{0}(t) \mathrm{d} t=\frac{1}{\Gamma(\alpha+1)} W(x),
$$

where

$$
W(x)=x^{\alpha} u(x)-(x-1)^{\alpha} u(x-1) .
$$

Equations (17) and (18) can be expanded into Haar wavelets as

$$
I^{\alpha} h_{n}(x)=c_{n 0} h_{0}(x)+\sum_{p=0}^{J} \sum_{q=0}^{2^{p}-1} c_{n 2^{p}+q} h_{2^{p}+q}(x)
$$

for $n=0,1, \ldots, m-1$

Now we want to obtain the coefficients $c_{n l}, n, l=$ $0,1, \ldots, m-1$ in the above equation. According to 4 and (5), we have

$$
\begin{aligned}
c_{00}= & \frac{1}{\Gamma(\alpha+1)} \int_{0}^{1} W(t) h_{0}(t) \mathrm{d} t=\frac{1}{\Gamma(\alpha+2)} \\
c_{02^{p}+q}= & \frac{2^{p}}{\Gamma(\alpha+1)} \int_{0}^{1} W(t) h_{2^{p}+q}(t) \mathrm{d} t \\
= & \frac{2^{p}}{\Gamma(\alpha+1)} \int_{0}^{1} W(t)\left\{u\left(t-\frac{q}{2^{p}}\right)\right. \\
& \left.-2 u\left(t-\frac{q+1 / 2}{2^{p}}\right)+u\left(t-\frac{q+1}{2^{p}}\right)\right\} \mathrm{d} t \\
= & \frac{2^{p}}{\Gamma(\alpha+1)}\left[\int_{\frac{q}{2^{p}}}^{1} t^{\alpha} \mathrm{d} t-2 \int_{\frac{q+1 / 2}{2^{p}}}^{t^{\alpha} \mathrm{d} t}\right. \\
& \left.+\int_{\frac{q+1}{2^{p}}}^{1} t^{\alpha} \mathrm{d} t\right] .
\end{aligned}
$$


Thus

$$
\begin{aligned}
c_{02^{p}}+q & \\
= & -\frac{2^{p}}{\Gamma(\alpha+2)} \\
& \cdot\left[\left(\frac{q}{2^{p}}\right)^{\alpha+1}-2\left(\frac{q+1 / 2}{2^{p}}\right)^{\alpha+1}+\left(\frac{q+1}{2^{p}}\right)^{\alpha+1}\right],
\end{aligned}
$$

where $p=0,1, \ldots, J \quad$ and $\quad q=0,1, \ldots, 2^{p}-1$.

Similarly, to calculate $c_{n 0}$ and $c_{n 2^{p}+q}$ for $n=$ $1,2, \ldots, m-1, p=0,1, \ldots, J$ and $q=0,1, \ldots, 2^{p}-1$ (in Eqn. (19)), we have

$$
\begin{aligned}
c_{n 0}= & \frac{1}{\Gamma(\alpha+1)} \int_{0}^{1}[X(t)-2 Y(t)+Z(t)] h_{0}(t) \mathrm{d} t \\
= & \frac{1}{\Gamma(\alpha+1)} \int_{0}^{1}[X(t)-2 Y(t)+Z(t)] \\
& \cdot[u(t)-u(t-1)] \mathrm{d} t \\
= & \frac{1}{\Gamma(\alpha+1)}\left[\int_{\frac{k}{2^{j}}}^{1}\left(t-\frac{k}{2^{j}}\right)^{\alpha} \mathrm{d} t\right. \\
& -2 \int_{\frac{k+1 / 2}{2^{j}}}^{1}\left(t-\frac{k+1 / 2}{2^{j}}\right)^{\alpha} \mathrm{d} t \\
& \left.+\int_{\frac{k+1}{2^{j}}}^{1}\left(t-\frac{k+1}{2^{j}}\right)^{\alpha} \mathrm{d} t\right] .
\end{aligned}
$$

Consequently,

$$
\begin{aligned}
c_{n 0} & \\
& =\frac{1}{\Gamma(\alpha+2)}\left[\left(1-\frac{k}{2^{j}}\right)^{\alpha+1}-2\left(1-\frac{k+1 / 2}{2^{j}}\right)^{\alpha+1}\right. \\
& \left.+\left(1-\frac{k+1}{2^{j}}\right)^{\alpha+1}\right] .
\end{aligned}
$$

and

$$
\begin{aligned}
& c_{n 2^{p}+q} \\
&=\frac{2^{p}}{\Gamma(\alpha+1)} \int_{0}^{1}[X(t)-2 Y(t)+Z(t)] h_{2^{p}+q}(t) \mathrm{d} t \\
&=\frac{2^{p}}{\Gamma(\alpha+1)} \int_{0}^{1}[X(t)-2 Y(t)+Z(t)]\left[u\left(t-\frac{q}{2^{p}}\right)\right. \\
&\left.-2 u\left(t-\frac{q+1 / 2}{2^{p}}\right)+u\left(t-\frac{q+1}{2^{p}}\right)\right] \mathrm{d} t \\
&= \frac{2^{p}}{\Gamma(\alpha+1)}\left[\int_{\eta_{0}}^{1}\left(t-\frac{k}{2^{j}}\right)^{\alpha} \mathrm{d} t\right.
\end{aligned}
$$

$$
\begin{aligned}
& \left.-2 \int_{\eta_{1 / 2}}^{1}\left(t-\frac{k}{2^{j}}\right)^{\alpha} \mathrm{d} t+\int_{\eta_{1}}^{1}\left(t-\frac{k}{2^{j}}\right)^{\alpha} \mathrm{d} t\right] \\
& -2 \frac{2^{p}}{\Gamma(\alpha+1)}\left[\int_{\theta_{0}}^{1}\left(t-\frac{k+1 / 2}{2^{j}}\right)^{\alpha} \mathrm{d} t\right. \\
& -2 \int_{\theta_{1 / 2}}^{1}\left(t-\frac{k+1 / 2}{2^{j}}\right)^{\alpha} \mathrm{d} t \\
& +\int_{\theta_{1}}^{1}\left(t-\frac{k+1 / 2}{2^{j}}\right)^{\alpha} \mathrm{d} t \\
& +\frac{2^{p}}{\Gamma(\alpha+1)}\left[\int_{\xi_{0}}^{1}\left(t-\frac{k+1}{2^{j}}\right)^{\alpha} \mathrm{d} t\right. \\
& -2 \int_{\xi_{1 / 2}}^{1}\left(t-\frac{k+1}{2^{j}}\right)^{\alpha} \mathrm{d} t+\int_{\xi_{1}}^{1}\left(t-\frac{k+1}{2^{j}}\right)^{\alpha} \mathrm{d} t,
\end{aligned}
$$

where

$$
\begin{array}{rlrl}
\eta_{i} & =\max \left\{\frac{k}{2^{j}}, \frac{q+i}{2^{p}}\right\}, & & i=0,1 / 2,1, \\
\theta_{i} & =\max \left\{\frac{k+1 / 2}{2^{j}}, \frac{q+i}{2^{p}}\right\}, & i=0,1 / 2,1, \\
\xi_{i} & =\max \left\{\frac{k+1}{2^{j}}, \frac{q+i}{2^{p}}\right\}, & i & i=0,1 / 2,1 .
\end{array}
$$

Therefore

$$
\begin{aligned}
c_{n 2^{p}}+q & -\frac{2^{p}}{\Gamma(\alpha+2)}\left[\left(\eta_{0}-\frac{k}{2^{j}}\right)^{\alpha+1}\right. \\
& \left.-2\left(\eta_{1 / 2}-\frac{k}{2^{j}}\right)^{\alpha+1}+\left(\eta_{1}-\frac{k}{2^{j}}\right)^{\alpha+1}\right] \\
& +2 \frac{2^{p}}{\Gamma(\alpha+2)}\left[\left(\theta_{0}-\frac{k+1 / 2}{2^{j}}\right)^{\alpha+1}\right. \\
& \left.-2\left(\theta_{1 / 2}-\frac{k+1 / 2}{2^{j}}\right)^{\alpha+1}+\left(\theta_{1}-\frac{k+1 / 2}{2^{j}}\right)^{\alpha+1}\right] \\
& -\frac{2^{p}}{\Gamma(\alpha+2)}\left[\left(\xi_{0}-\frac{k+1}{2^{j}}\right)^{\alpha+1}\right. \\
& \left.-2\left(\xi_{1 / 2}-\frac{k+1}{2^{j}}\right)^{\alpha+1}+\left(\xi_{1}-\frac{k+1}{2^{j}}\right)^{\alpha+1}\right],
\end{aligned}
$$

for $n=1,2, \ldots, m-1, p=0,1, \ldots, J$ and $q=$ $0,1, \ldots, 2^{p}-1$. Thus, we can write the operational matrix of fractional integration as

$$
\mathbf{P}_{m}^{\alpha}=\left[\begin{array}{cc}
\mathbf{P}_{m / 2}^{\alpha} & \mathbf{R}_{m / 2 \times m / 2} \\
\mathbf{S}_{m / 2 \times m / 2} & \mathbf{U}_{m / 2 \times m / 2}
\end{array}\right],
$$

where

$$
\mathbf{R}_{m / 2 \times m / 2}=\left[c_{n 2^{J}+q}\right],
$$

for $n=0,1, \ldots, m / 2-1, q=0,1, \ldots, 2^{J}-1$ and $\mathbf{S}_{m / 2 \times m / 2}=\left[c_{n l}\right], n=m / 2, \ldots, m-1$, and $l=$ $0,1, \ldots, m / 2-1$ are calculated easily by (20), 22) and (21), 22), respectively, and $\mathbf{U}_{m / 2 \times m / 2}$ is an upper triangular matrix in the form

$\mathbf{U}_{m / 2 \times m / 2}=u_{1} \mathbf{I}+u_{2} \mu+u_{3} \mu^{2}+\cdots+u_{m / 2} \mu^{m / 2-1}$, 
such that

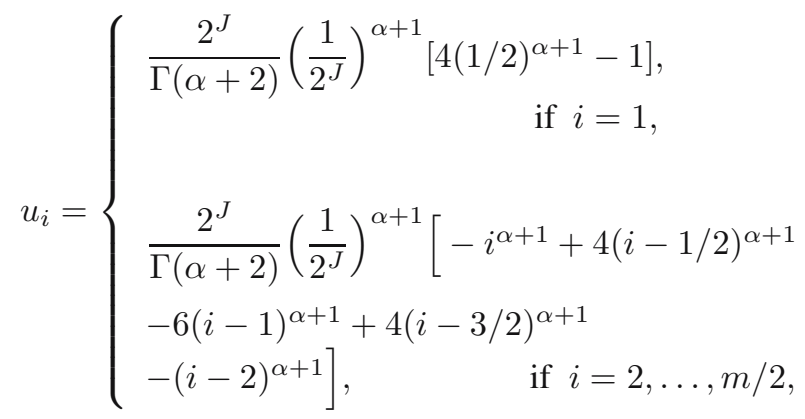

$\mathbf{I}_{m / 2}$ is an $(m / 2) \times(m / 2)$ identity matrix and

$$
\mu_{m / 2}=\left[\begin{array}{ccccc}
0 & 1 & 0 & \ldots & 0 \\
0 & 0 & 1 & \ddots & \vdots \\
\vdots & & \ddots & \ddots & 0 \\
& & & & 1 \\
0 & & & \ldots & 0
\end{array}\right]_{m / 2 \times m / 2}
$$

It is essential that, for $\alpha=1$, the fractional integration (10) is the ordinary integration and the generalized operational matrix of fractional integration $\mathbf{P}_{m}^{\alpha}$ is the same as $\mathbf{P}_{m}$, which is introduced in (12). Here we present $\mathbf{P}_{m}^{1 / 3}$ for $J=0,1$ :

$$
\begin{aligned}
\mathbf{P}_{2}^{1 / 3}= & {\left[\begin{array}{cc}
0.8399 & -0.1733 \\
0.1733 & 0.4933
\end{array}\right], } \\
\mathbf{P}_{4}^{1 / 3}= & {\left[\begin{array}{cccc}
0.8399 & -0.1733 & -0.1375 & -0.0571 \\
0.1733 & 0.4933 & -0.1375 & 0.2179 \\
0.0286 & 0.1090 & 0.3916 & -0.0428 \\
0.0688 & -0.0688 & 0 & 0.3916
\end{array}\right], }
\end{aligned}
$$

\section{Application of the method}

According to Section (2), the right-hand side of Eqn. (1) is approximated as

$$
g(x) \cong g_{0} h_{0}(x)+\sum_{j=0}^{J} \sum_{k=0}^{2^{j}-1} g_{2^{j}+k} h_{2^{j}+k}(x)=\mathbf{g}^{T} \mathbf{h}(x) .
$$

Similarly, $K(x, t) \in L^{2}([0,1) \times[0,1))$ can be approximated as

$$
k(x, t) \cong \sum_{i=0}^{m-1} \sum_{j=0}^{m-1} k_{i j} h_{i}(x) h_{j}(t)
$$

or, in matrix form,

$$
k(x, t) \cong \mathbf{h}^{T}(x) \mathbf{K h}(t),
$$

where $\mathbf{K}=\left[k_{i j}\right]_{m \times m}$, such that

$$
\begin{aligned}
& k_{i j}=2^{i+j} \int_{0}^{1} \int_{0}^{1} k(x, t) h_{i}(x) h_{j}(t) \mathrm{d} t \mathrm{~d} x, \\
& i, j=0,1, \ldots, m-1 .
\end{aligned}
$$

Also, the fractional integral part of (1) is written as

$$
\frac{1}{\Gamma(\alpha)} \int_{0}^{x}(x-t)^{\alpha-1} k(x, t) f(t) \mathrm{d} t \cong \widetilde{\mathbf{v}} \mathbf{h}(x) .
$$

The details of calculating $\widetilde{\mathbf{v}}$ are shown in Appendix. By substituting the approximations (9), (33) and (22) into (1), we obtain

$$
\mathbf{h}^{T}(x) \mathbf{c}-\mathbf{h}^{T}(x) \widetilde{\mathbf{v}}=\mathbf{h}^{T}(x) \mathbf{g} .
$$

Therefore,

$$
\mathbf{c}-\widetilde{\mathbf{v}}=\mathbf{g} .
$$

Equation (25) is a system of linear equations and can be easily solved for the unknown vector c. Note that the entries of the vector $\widetilde{\mathbf{v}}$ are related to the entries of $\mathbf{c}$. Similarly, the Abel integral equation (2) can be written as

$$
\mathbf{h}^{T}(x) \mathbf{c}-\mathbf{h}^{T}(x)\left(\mathbf{P}_{m}^{\alpha}\right)^{T} \mathbf{c}=\mathbf{h}^{T}(x) \mathbf{g} .
$$

Therefore,

$$
\mathbf{c}-\left(\mathbf{P}_{m}^{\alpha}\right)^{T} \mathbf{c}=\mathbf{g} .
$$

Equation (26) is a system of linear equations and can be easily solved for the unknown vector $\mathbf{c}$ :

$$
\mathbf{c}=\left(I-\left(\mathbf{P}_{m}^{\alpha}\right)^{T}\right)^{-1} \mathbf{g}
$$

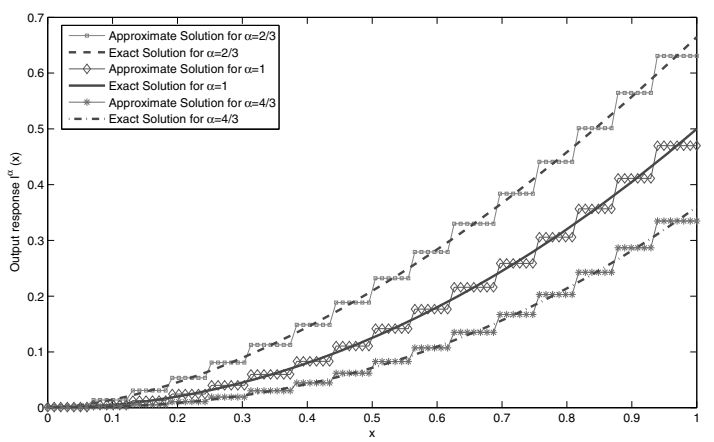

Fig. 1. Fractional integration of $f(x)=x$ and its approximation $\left(I^{\alpha} f_{16}(x)\right)$ for $\alpha=2 / 3,1,4 / 3$.

\section{Error analysis}

Let us consider the following integral equation:

$$
\begin{array}{r}
f(x)-\frac{1}{\Gamma(\alpha)} \int_{0}^{x}(x-t)^{\alpha-1} k(x, t) f(t) \mathrm{d} t=g(x), \\
0 \leq x \leq 1 .
\end{array}
$$

Suppose that $f(x)$ is the exact solution of the above integral equation. In order to analyze the convergence of our method, we will define the following error function and show that the method is convergent for a special class of functions in the sense that the corresponding error tends to zero as $m$ tends to infinity. 
Definition 1. If $f(x)$ and $f_{m}(x)=\mathbf{c}^{T} \mathbf{h}(x)$ are the exact and approximate solutions of 27, respectively, then the corresponding error is defined as follows:

It is clear that

$$
e_{m}(x)=f(x)-f_{m}(x) .
$$

$$
e_{m}(x)=\sum_{j=J+1}^{\infty} \sum_{k=0}^{2^{j}-1} c_{2^{j}+k} h_{2^{j}+k}(x) .
$$

Now we will prove the following convergence theorem.

Theorem 1. Suppose that $f(x)$ satisfies a Lipschitz condition on $[0,1]$, that is,

$$
\exists M>0, \forall x, y \in[0,1]:|f(x)-f(y)| \leq M|x-y| .
$$

Then the Haar wavelet method will be convergent in the sense that $e_{m}(x)$ goes to zero as $m$ goes to infinity. Moreover, the convergence is of order one, that is,

$$
\left\|e_{m}(x)\right\|_{2}=O\left(\frac{1}{m}\right)
$$

Proof. We have

$$
\begin{aligned}
\left\|e_{m}(x)\right\|_{2}^{2}= & \int_{0}^{1}\left(\sum_{j=J+1}^{\infty} \sum_{k=0}^{2^{j}-1} c_{2^{j}+k} h_{2^{j}+k}(x)\right)^{2} \mathrm{~d} x \\
= & \sum_{j=J+1}^{\infty} \sum_{k=0}^{2^{j}-1} c_{2^{j}+k}^{2} \int_{0}^{1} h_{2^{j}+k}^{2}(x) \mathrm{d} x \\
& +\sum_{j=J+1}^{\infty} \sum_{k=0}^{2^{j}-1} \sum_{p=J+1}^{\infty} \sum_{q=0, q \neq k}^{2^{p}-1}\left\{c_{2^{j}+k}\right. \\
& \left.\cdot c_{2^{p}+q} \int_{0}^{1} h_{2^{j}+k}(x) h_{2^{p}+q}(x)\right\} \mathrm{d} x \\
= & \sum_{j=J+1}^{\infty} \sum_{k=0}^{2^{j}-1} c_{2^{j}+k}^{2}\left(\frac{1}{2^{j}}\right) .
\end{aligned}
$$

Since $c_{2^{j}+k}=2^{j} \int_{0}^{1} f(x) h_{i}(x) \mathrm{d} x$, by 18 and using the mean value theorem, we have

$$
\exists x_{1}^{j k} \in\left[\frac{k}{2^{j}}, \frac{k+1 / 2}{2^{j}}\right], x_{2}^{j k} \in\left[\frac{k+1 / 2}{2^{j}}, \frac{k+1}{2^{j}}\right],
$$

such that

$$
\begin{aligned}
c_{2^{j}+k}= & 2^{j}\left[\left(\frac{k+1 / 2}{2^{j}}-\frac{k}{2^{j}}\right) f\left(x_{1}^{j k}\right)\right. \\
& \left.-\left(\frac{k+1}{2^{j}}-\frac{k+1 / 2}{2^{j}}\right) f\left(x_{2}^{j k}\right)\right] \\
= & \frac{1}{2}\left[f\left(x_{1}^{j k}\right)-f\left(x_{2}^{j k}\right)\right] \\
\leq & \frac{1}{2} M\left(x_{1}^{j k}-x_{2}^{j k}\right) \\
\leq & \frac{1}{2} M \frac{1}{2^{j}}=M \frac{1}{2^{j+1}} .
\end{aligned}
$$

The first inequality is obtained by (28). Therefore, $c_{2^{j}+k}^{2} \leq M^{2} \frac{1}{2^{2 j+2}}$ and

$$
\begin{aligned}
\left\|e_{m}(x)\right\|_{2}^{2} & =\sum_{j=J+1}^{\infty} \sum_{k=0}^{2^{j}-1} c_{2^{j}+k}^{2}\left(\frac{1}{2^{j}}\right) \\
& \leq \sum_{j=J+1}^{\infty} \sum_{k=0}^{2^{j}-1} M^{2} \frac{1}{2^{2 j+2}}\left(\frac{1}{2^{j}}\right) \\
& =\frac{M^{2}}{4} \sum_{j=J+1}^{\infty} 2^{j} \frac{1}{2^{3 j}} \\
& =\frac{M^{2}}{3}\left(\frac{1}{2^{J+1}}\right)^{2} .
\end{aligned}
$$

Since $m=2^{J+1}$, we have

$$
\left\|e_{m}(x)\right\|_{2}^{2} \leq \frac{M^{2}}{3}\left(\frac{1}{m}\right)^{2}
$$

or, in other words,

$$
\left\|e_{m}(x)\right\|_{2}=O\left(\frac{1}{m}\right)
$$

By the above proof, we can obtain a bound for $\left\|e_{m}(x)\right\|_{2}$

$$
\left\|e_{m}(x)\right\|_{2} \leq \frac{M}{m \sqrt{3}}
$$

At the end of this section, we discuss the conditions under which our method will fail or will not give us an acceptable approximation. The solution $f(x)$ of Eqn. (27) is generally not differentiable at the initial point (Vainikko and Pedas, 1981). On the other hand, the Lipschitz continuity of functions on the real line is closely related to differentiability, i.e., an everywhere differentiable function $f: \mathbb{R} \rightarrow \mathbb{R}$ is Lipschitz continuous (with $\left.M=\sup \left|f^{\prime}(x)\right|\right)$ if and only if it has the first derivative bounded (one direction follows from the mean value theorem). So due to the non-smoothness of $f(x)$ at the initial point, the Lipschitz constant $M$ may not exist or may be too large. Therefore, the error bound 29) will increase and we will not have a good approximation.

5.1. Estimation of the error function. In real problems, we often tend to solve equations with unknown exact solutions. These unknown exact solutions may be singular, smooth or not. Hence, when we apply our method to these problems, we cannot say that this approximate solution is good or bad unless we are able to calculate the error function $e_{m}(x)$. Therefore, it is necessary to introduce a process for estimating the error function when the exact solution is unknown. 

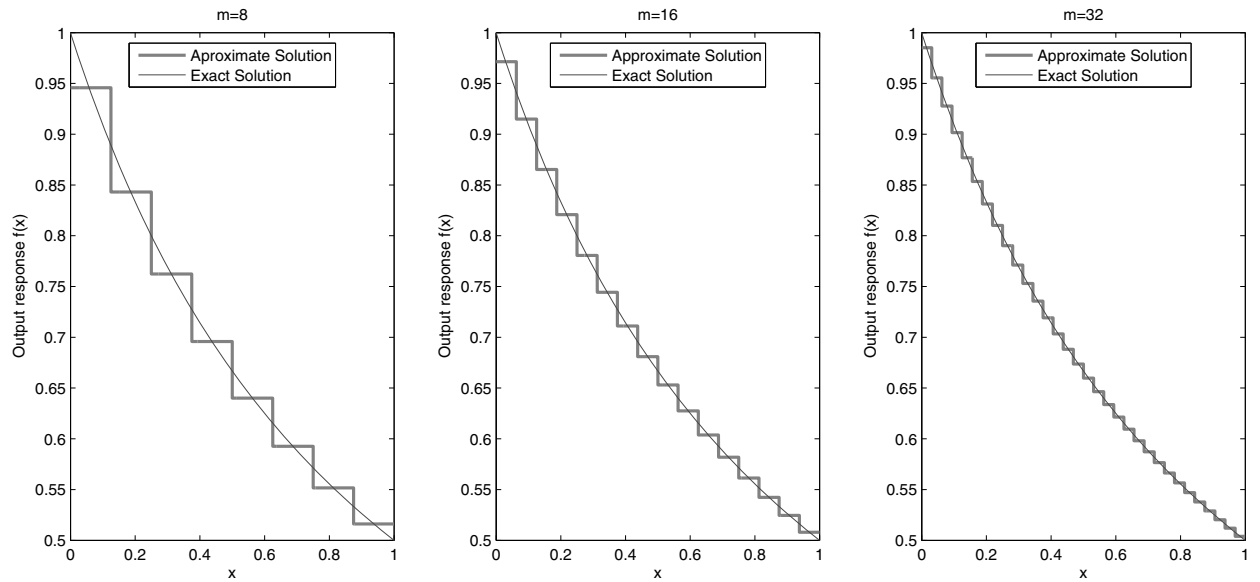

Fig. 2. Approximate solutions of Example 2 for selected values of $m$.
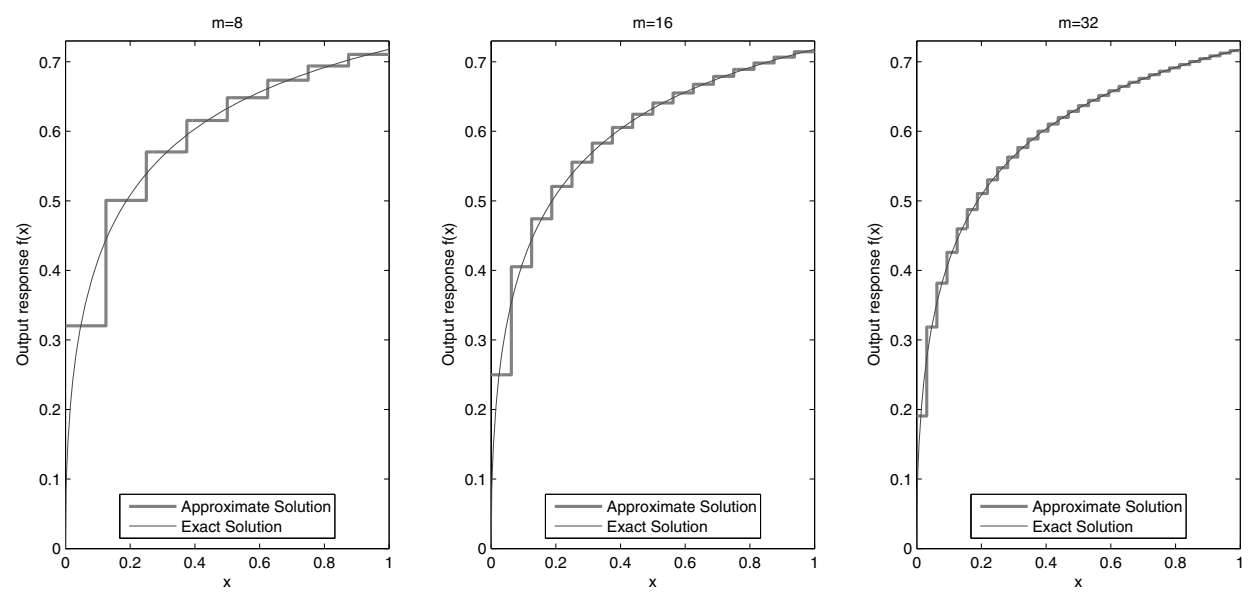

Fig. 3. Approximate solutions of Example 3 for selected values of $m$.

Here we introduce a method to estimate the error function. Since $f_{m}(x)$ is considered an approximate solution of Eqn. (1), it satisfies the following equation:

$$
\begin{array}{r}
f_{m}(x)-\frac{1}{\Gamma(\alpha)} \int_{0}^{x}(x-t)^{\alpha-1} k(x, t) f_{m}(t) \mathrm{d} t \\
=g(x)+r_{m}(x),
\end{array}
$$

The perturbation term $r_{m}(x)$ can be obtained by substituting the estimated solution $f_{m}(x)$ into the equation

$$
\begin{aligned}
r_{m} & (x) \\
= & f_{m}(x)-\frac{1}{\Gamma(\alpha)} \int_{0}^{x}(x-t)^{\alpha-1} k(x, t) f_{m}(t) \mathrm{d} t \\
& -g(x) .
\end{aligned}
$$

Subtracting (30) from (1), we get the following equation: $e_{m}(x)-\frac{1}{\Gamma(\alpha)} \int_{0}^{x}(x-t)^{\alpha-1} k(x, t) e_{m}(t) \mathrm{d} t=-r_{m}(x)$.
Obviously, the above is a fractional Volterra integral equation in which the error function, $e_{m}(x)$, is unknown. We can easily apply our method to the above equation to find an approximation of the error function $e_{m}(x)$.

\section{Numerical examples}

To show the efficiency of the proposed method, we will apply our method to obtain the approximate solution of the following examples. All of the computations have been performed using MATLAB 7.8. Note that

$$
\begin{aligned}
\left\|e_{m}(x)\right\|_{2} & =\left(\int_{0}^{1} e_{m}^{2}(x) \mathrm{d} x\right)^{1 / 2} \\
& \cong\left(\frac{1}{N} \sum_{i=0}^{N} e_{m}^{2}\left(x_{i}\right)\right)^{1 / 2},
\end{aligned}
$$

where $f(x)$ is the exact solution and $f_{m}(x)$ is the approximate solution obtained by Eqn. (99). 
Example 1. Let $f(x)=x$. Here we approximate $I^{\alpha} f(x)$ by $\mathbf{P}_{m}^{\alpha}$ for $\alpha=2 / 3,1,4 / 3$ and compare it with the exact fractional integration of the function $f(x)=x$, which is easily obtained by (11). If we write $x \cong \mathbf{c}^{T} \mathbf{h}(x)$, we will have $I^{\alpha} x \cong \mathbf{c}^{T} I^{\alpha} \mathbf{h}(x) \cong \mathbf{c}^{T} \mathbf{P}_{m}^{\alpha} \mathbf{h}(x)$. Numerical results for $m=16$ are shown in Fig. 1

Example 2. (Pandey et al., 2009) Consider the following Abel integral equation of the second kind:

$$
\begin{aligned}
f(x)= & \frac{1}{1+x}+\frac{2 \operatorname{arcsinh}(\sqrt{x})}{\sqrt{1+x}} \\
& -\int_{0}^{x} \frac{f(t)}{\sqrt{x-t}} \mathrm{~d} t=x, \quad 0 \leq x \leq 1,
\end{aligned}
$$

which has the exact solution $f(x)=1 /(1+x)$. Numerical results are shown in Table 1 and Fig. 2.

Example 3. (Yousefi, 2006) Consider the following Abel integral equation of the second kind:

$$
f(x)=2 \sqrt{x}-\int_{0}^{x} \frac{f(t)}{\sqrt{x-t}} \mathrm{~d} t=x, \quad 0 \leq x \leq 1,
$$

which has $f(x)=1-e^{\pi x} \operatorname{erfc}(\sqrt{\pi x})$ as the exact solution, where the complementary error function $\operatorname{erfc}(x)$ is defined as

$$
\operatorname{erfc}(x)=\frac{2}{\sqrt{\pi}} \int_{x}^{\infty} e^{-t^{2}} \mathrm{~d} t
$$

Here $\alpha=1 / 2$. Numerical results are shown in Table 1 and Fig. 3.

Example 4. Consider the following fractional Volterra integral equation:

$$
f(x)+\int_{0}^{x} \frac{x t}{\sqrt{x-t}} f(t) \mathrm{d} t=g(x), \quad 0 \leq x \leq 1,
$$

where

$$
g(x)=x(1-x)+\frac{16}{105} x^{\frac{7}{2}}(7-6 x)
$$

and $f(x)=x(1-x)$ is the exact solution. The numerical results are shown in Table 1 and Fig. 4.

Let us consider examples with nonsmooth and singular solutions.

Example 5. (Pandey, 2009) Consider the fractional Volterra integral equation:

$$
f(x)=\frac{1}{\sqrt{x}}+\pi-\int_{0}^{x} \frac{f(t)}{\sqrt{x-t}} \mathrm{~d} t=x, \quad 0 \leq x \leq 1,
$$

which has $f(x)=1 / \sqrt{x}$ as the exact solution. In this case there is a singularity at the point $x=0$. As discussed in Section 5, the solution around this point is not so good (see Fig. 5). Hence the numerical results in Table 1 have been calculated in $[0.1,1]$. As can be seen, our method provides reasonable estimates even in this case with a singular solution.
Example 6. Consider the following Abel integral equation of the first kind:

$$
\int_{0}^{x} \frac{f(t)}{\sqrt{x-t}} \mathrm{~d} t=x
$$

with the exact solution $f(x)=\frac{2}{\pi} \sqrt{x}$. Numerical results are shown in Fig. 6 and Table 1, for some selected values of $m$.

Example 7. (Abdalkhania, 1990; Dixon, 1985) Consider the following fractional Volterra integral equation:

$$
f(x)+\int_{0}^{x} \frac{f(t)}{\sqrt{x-t}} \mathrm{~d} t=\frac{1}{2} \pi x+\sqrt{x}, \quad 0 \leq x \leq 1,
$$

which has $f(x)=\sqrt{x}$ as the exact solution. Numerical results are shown in Table 1 and Fig. 7.

As can be seen, in contrast to the singular solution (Example 6), the method provides rather accurate results for nonsmooth solutions (Examples 6 and 7).

\section{Conclusion}

Haar wavelets have been applied to study integral equations with a nonsingular kernel (Lepik, 2004; Maleknejad and Mirzaee, 2005). In this paper we use them to solve the fractional Volterra integral equation which has a weakly singular kernel. For this purpose, we generalized the operational matrix of integration to the case of fractional integration. We considered various types of solutions, with smooth, nonsmooth and even singular behaviors. In all the cases considered, the method provides reasonable estimates. Numerical examples and their error analysis show that more accurate results are obtained when finer resolutions are used. We hope the method to be generalized to the case of fractional Fredholm integral equations, which is the interest of current applications in computational chemistry (Chiodo, 2007; Chuev, 2006; 2007; 2008).

\section{Acknowledgment}

The authors are thankful to Prof. M.V. Fedorov for his support, and they also acknowledge the Max Planck Institute for Mathematics in the Sciences (MPI MIS, Leipzig). G.N. Chuev is thankful for a partial support within the Marie Curie Fellowship (PIIF-GA-2009-235064). M. Mohseni Moghadam is thankful for a partial support from the Mahani Mathematical Research Center of the Shahid Bahonar University of Kerman. This work has also been partially supported by the Center of Excellence in Linear Algebra and Optimization of the Shahid Bahonar University of Kerman. 

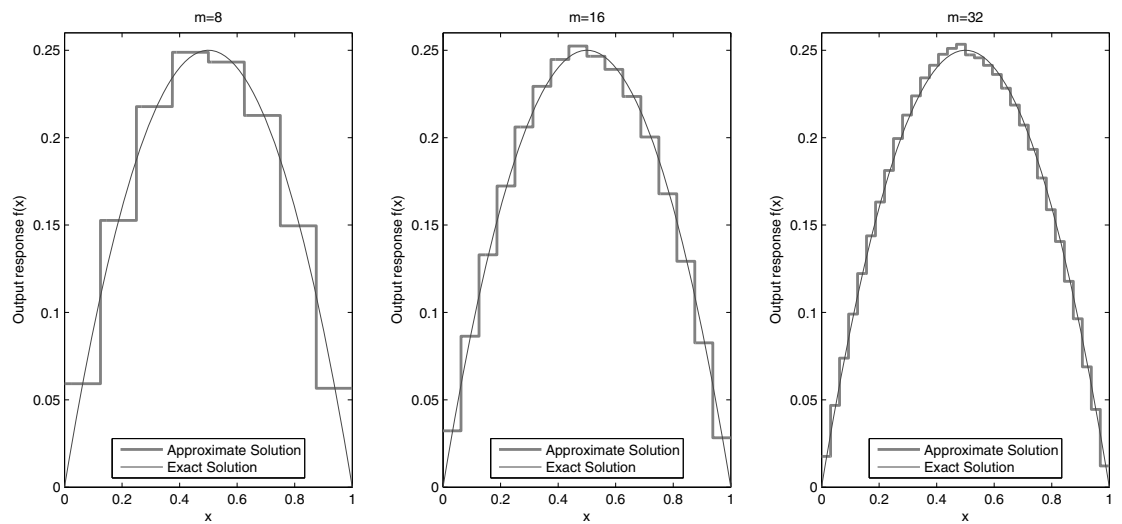

Fig. 4. Approximate solutions of Example 4 for selected values of $m$.

Table 1. Approximate norm-2 of the absolute error, $\left\|e_{m}(x)\right\|_{2}$, for $m=8,16,32,64,128$.

\begin{tabular}{|c|c|c|c|c|c|}
\hline Examples & $\left\|e_{8}(x)\right\|_{2}$ & $\left\|e_{16}(x)\right\|_{2}$ & $\left\|e_{32}(x)\right\|_{2}$ & $\left\|e_{64}(x)\right\|_{2}$ & $\left\|e_{128}(x)\right\|_{2}$ \\
\hline \hline Example 2 & $3.7602 \mathrm{e}+000$ & $9.5290 \mathrm{e}-005$ & $2.3838 \mathrm{e}-005$ & $5.9609 \mathrm{e}-006$ & $1.4936 \mathrm{e}-006$ \\
Example 3 & $1.3111 \mathrm{e}-003$ & $4.8933 \mathrm{e}-004$ & $1.7606 \mathrm{e}-004$ & $6.1280 \mathrm{e}-005$ & $2.1571 \mathrm{e}-005$ \\
Example 4 & $4.3628 \mathrm{e}-004$ & $1.1572 \mathrm{e}-004$ & $3.4342 \mathrm{e}-005$ & $1.4039 \mathrm{e}-005$ & $9.0411 \mathrm{e}-006$ \\
Example 5 & $1.2696 \mathrm{e}+001$ & $3.7150 \mathrm{e}-003$ & $1.0089 \mathrm{e}-003$ & $2.6852 \mathrm{e}-004$ & $8.4154 \mathrm{e}-005$ \\
Example 6 & $6.9314 \mathrm{e}-004$ & $1.9918 \mathrm{e}-004$ & $5.7015 \mathrm{e}-005$ & $1.6171 \mathrm{e}-005$ & $4.6829 \mathrm{e}-006$ \\
Example 7 & $1.5713 \mathrm{e}-003$ & $4.5647 \mathrm{e}-004$ & $1.3244 \mathrm{e}-004$ & $3.8332 \mathrm{e}-005$ & $1.1353 \mathrm{e}-005$ \\
\hline
\end{tabular}
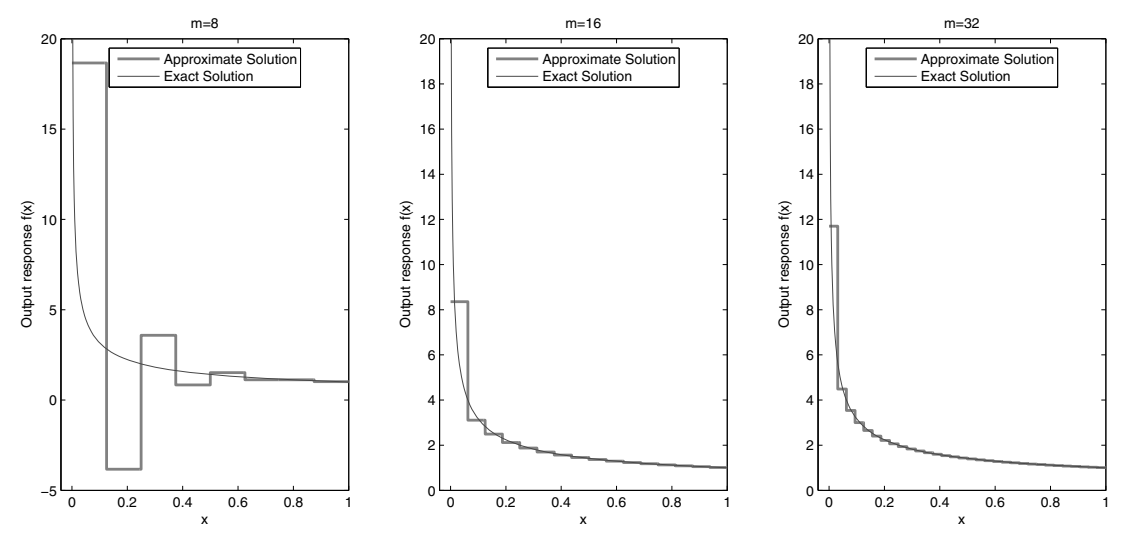

Fig. 5. Approximate solutions of Example 5 for selected values of $m$.
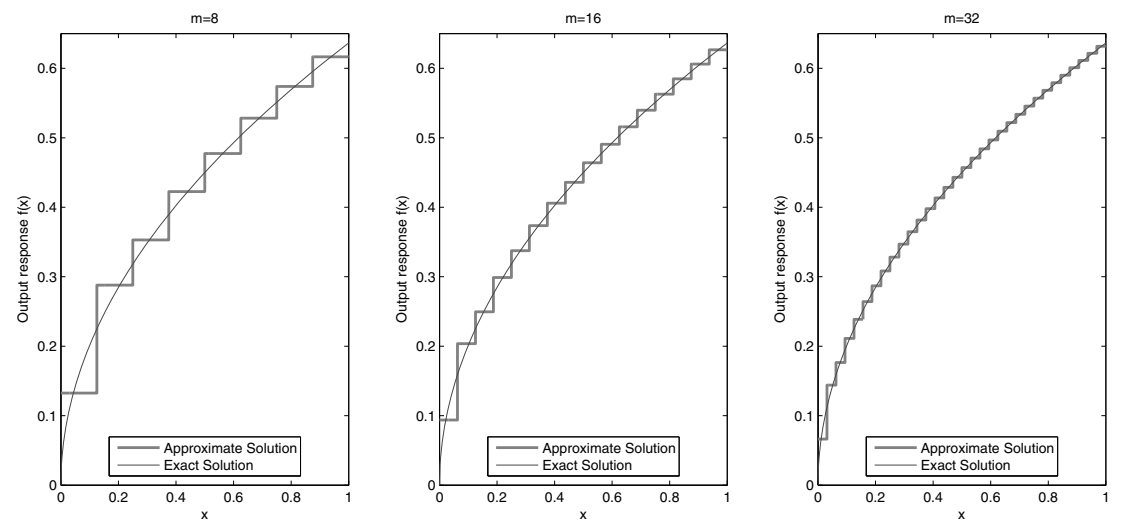

Fig. 6. Approximate solutions of Example 6 for selected values of $m$. 

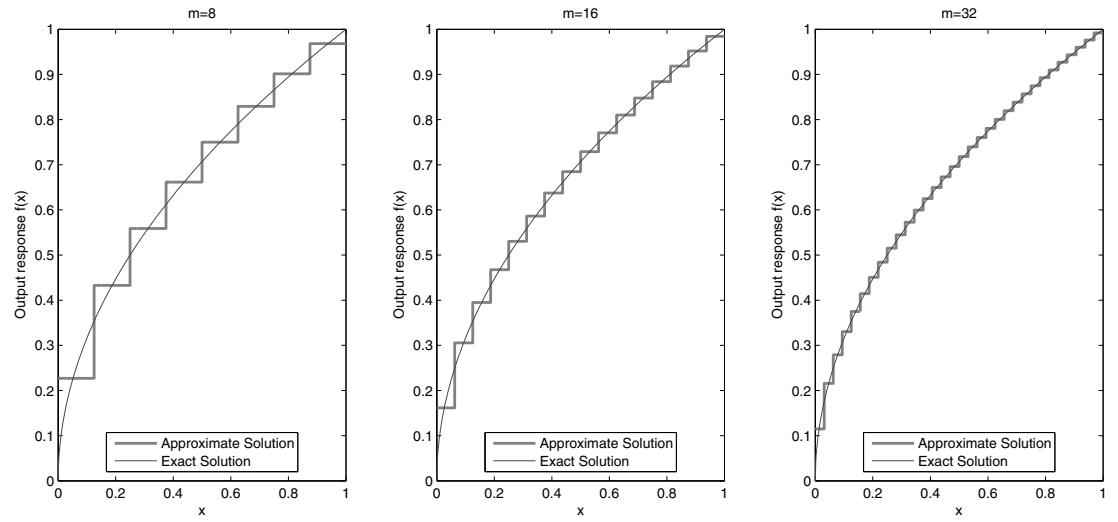

Fig. 7. Approximate solutions of Example 7 for selected values of $m$.

\section{References}

Abdalkhania, J. (1990). Numerical approach to the solution of Abel integral equations of the second kind with nonsmooth solution, Journal of Computational and Applied Mathematics 29(3): 249-255.

Akansu, A.N. and Haddad, R.A. (1981). Multiresolution Signal Decomposition, Academic Press Inc., San Diego, CA

Bagley, R.L. and Torvik, P.J. (1985). Fractional calculus in the transient analysis of viscoelastically damped structures, American Institute of Aeronautics and Astronautics Journal 23(6): 918-925.

Baillie, R.T. (1996). Long memory processes and fractional integration in econometrics, Journal of Econometrics 73(1): 559.

Baratella, P. and Orsi, A.P. (2004). New approach to the numerical solution of weakly singular Volterra integral equations, Journal of Computational and Applied Mathematics 163(2): 401-418.

Brunner, H. (1984). The numerical solution of integral equations with weakly singular kernels, in D.F. GriMths (Ed.), Numerical Analysis, Lecture Notes in Mathematics, Vol. 1066, Springer, Berlin, pp. 50-71.

Chen, C.F. and Hsiao, C.H. (1997). Haar wavelet method for solving lumped and distributed parameter systems, IEE Proceedings: Control Theory and Applications 144(1): 87-94.

Chena, W., Suna, H., Zhang, X. and Korŏsak, D. (2010). Anomalous diffusion modeling by fractal and fractional derivatives, Computers \& Mathematics with Applications 59(5): 265-274.

Chiodo, S., Chuev, G.N., Erofeeva, S.E., Fedorov, M.V., Russo, N. and Sicilia, E. (2007). Comparative study of electrostatic solvent response by RISM and PCM methods, International Journal of Quantum Chemistry 107: 265-274.

Chow, T.S. (2005). Fractional dynamics of interfaces between soft-nanoparticles and rough substrates, Physics Letters A 342(1-2): 148-155.

Chuev, G.N., Fedorov, M.V. and Crain, J. (2007). Improved estimates for hydration free energy obtained by the reference interaction site model, Chemical Physics Letters 448: 198 202.

Chuev, G.N., Fedorov, M.V., Chiodo, S., Russo, N. and Sicilia, E. (2008). Hydration of ionic species studied by the reference interaction site model with a repulsive bridge correction, Journal of Computational Chemistry 29(14): 24062415.

Chuev, G.N., Chiodo, S., Fedorov, M.V., Russo, N. and Sicilia, E. (2006). Quasilinear RISM-SCF approach for computing solvation free energy of molecular ions, Chemical Physics Letters 418: 485-489.

Dixon, J. (1985). On the order of the error in discretization methods for weakly singular second kind Volterra integral equations with non-smooth solution, BIT 25(4): 624-634.

Hsiao, C.H. and Wu, S.P. (2007). Numerical solution of timevarying functional differential equations via Haar wavelets, Applied Mathematics and Computation 188(1): 1049 1058.

Lepik, Ü. and Tamme, E. (2004). Application of the Haar wavelets for solution of linear integral equations, $D y$ namical Systems and Applications, Proceedings, Antalya, Turkey, pp. 494-507.

Lepik, Ü. (2009). Solving fractional integral equations by the Haar wavelet method, Applied Mathematics and Computation 214(2): 468-478.

Li, C. and Wang, Y. (2009). Numerical algorithm based on Adomian decomposition for fractional differential equations, Computers \& Mathematics with Applications 57(10): 1672-1681.

Magin, R.L. (2004). Fractional calculus in bioengineering. Part 2, Critical Reviews in Bioengineering 32: 105-193.

Mainardi, F. (1997). Fractional calculus: 'Some basic problems in continuum and statistical mechanics', in A. Carpinteri and F. Mainardi (Eds.), Fractals and Fractional Calculus in Continuum Mechanics, Springer-Verlag, New York, NY.

Mandelbrot, B. (1967). Some noises with 1/f spectrum, a bridge between direct current and white noise, IEEE Transactions on Information Theory 13: 289-298. 
Maleknejad, K. and Mirzaee, F. (2005). Using rationalized Haar wavelet for solving linear integral equations, Applied Mathematics and Computation 160(2): 579-587.

Meral, F.C., Royston, T.J. and Magin, R. (2010). Fractional calculus in viscoelasticity: An experimental study, Соттиnications in Nonlinear Science and Numerical Simulation 15(4): 939-945.

Metzler, R. and Nonnenmacher, T.F. (2003). Fractional relaxation processes and fractional rheological models for the description of a class of viscoelastic materials, International Journal of Plasticity 19(7): 941-959.

Miller, K. and Feldstein, A. (1971). Smoothness of solutions of Volterra integral equations with weakly singular kernels, SIAM Journal on Mathematical Analysis 2: 242-258.

Miller, K. and Ross, B. (1993). An Introduction to the Fractional Calculus and Fractional Differential Equations, John Wiley and Sons, New York, NY.

Pandey, R.K., Singh, O.P. and Singh, V.K. (2009). Efficient algorithms to solve singular integral equations of Abel type, Computers and Mathematics with Applications 57(4): 664-676.

Podlubny, I. (1999). Fractional Differential Equations, Academic Press, New York, NY.

Strang, G. (1989). Wavelets and dilation equations, SIAM Review 31(4): 614-627.

Vainikko, G. and Pedas, A. (1981). The properties of solutions of weakly singular integral equations, Journal of the Australian Mathematical Society, Series B: Applied Mathematics 22: 419-430.

Vetterli, M. and Kovacevic, J. (1995). Wavelets and Subband Coding, Prentice Hall, Englewood Cliffs, NJ.

Yousefi, S.A. (2006). Numerical solution of Abel's integral equation by using Legendre wavelets, Applied Mathematics and Computation 175(1): 574-580.

Zaman, K.B.M.Q. and Yu, J.C. (1995). Power spectral density of subsonic jetnoise, Journal of Sound and Vibration 98(4): 519-537.

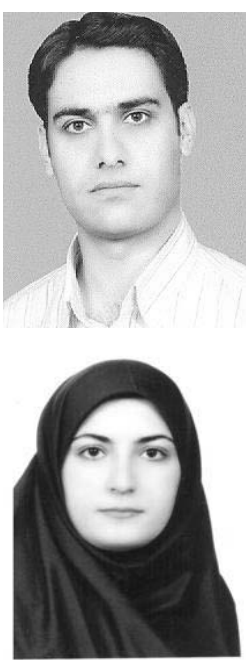

Habibollah Saeedi was born in Shahreza, Isfahan, Iran, in 1981. He obtained his M.Sc. (2005) and Ph.D. (2011) degrees in numerical analysis from the Shahid Bahonar University of Kerman, Iran. His research interests include numerical solution of equations and numerical linear algebra.

Nasibeh Mollahasani was born in Kerman, Iran, in 1983. She obtained her B.Sc. in pure mathematics, 2005, from the Shahid Bahonar University of Kerman, Iran, and her M.Sc. in numerical analysis in 2007 from the same university. Her research interests include numerical solution of equations and numerical linear algebra.

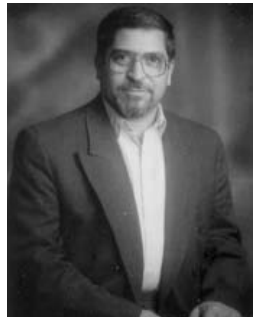

Moamoud Mohseni Moghadam was born in Rafsanjan, Iran, in 1948. He received his M.Sc. and Ph.D. degrees in applied mathematics from the Michigan State University, E-Lansing, MI, USA, in 1984. Currently, he is a professor of mathematics at the Shahid Bahonar University of Kerman, Iran. His research interests include numerical solutions of equations and numerical linear algebra.

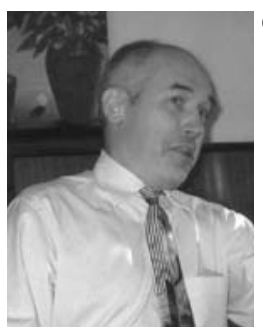

Gennady N. Chuev was born in Grozny, Russia, in 1961. He graduated from Moscow Physical Technical Institute in 1984. He received his Ph.D. (1990) and Doctor (2001) degrees in physics. In 2002 he was appointed the leading scientist at the Institute of Theoretical and Experimental Biophysics of the Russian Academy of Sciences (Pushchino). His field of specialization is computational chemical physics, while his primary interests focus on solvation effects and applications of multi-scale methods to investigate these phenomena.

\section{Appendix}

\section{Evaluating $\widetilde{\mathbf{v}}$}

The fractional integral part of (1) can be written as

$$
\frac{1}{\Gamma(\alpha)} \int_{0}^{x}(x-t)^{\alpha-1} k(x, t) f(t) \mathrm{d} t \cong \widetilde{\mathbf{v}} \mathbf{h}(x),
$$

where $\widetilde{\mathbf{v}}=\left[\widetilde{v}_{0}, \widetilde{v}_{1}, \ldots, \widetilde{v}_{m-1}\right]^{T}$. According to Eqn. (7), we have

$$
\begin{aligned}
& \widetilde{v}_{i} \\
& =2^{j} \int_{0}^{1}\left[\frac{1}{\Gamma(\alpha)} \int_{0}^{x}(x-t)^{\alpha-1} k(x, t) f(t) \mathrm{d} t\right] h_{i}(x) \mathrm{d} x,
\end{aligned}
$$

for $i=2^{j}+k$. Substituting $f(t) \cong \mathbf{h}^{T}(t) \mathbf{c}$ and (23) into Eqn. (34), we obtain

$\widetilde{v}_{i}$

$$
\begin{aligned}
\cong & 2^{j} \int_{0}^{1}\left[\frac{1}{\Gamma(\alpha)} \int_{0}^{x}(x-t)^{\alpha-1} \mathbf{h}^{T}(x) \mathbf{K h}(t) \mathbf{h}^{T}(t) \mathbf{c} \mathrm{d} t\right] \\
& \cdot h_{i}(x) \mathrm{d} x \\
= & 2^{j} \int_{0}^{1} \mathbf{h}^{T}(x) \mathbf{K} \mathbf{M}\left[\frac{1}{\Gamma(\alpha)} \int_{0}^{x}(x-t)^{\alpha-1} \mathbf{h}(t) \mathrm{d} t\right] \\
& \cdot h_{i}(x) \mathrm{d} x,
\end{aligned}
$$

where the $m \times m$ matrix $\mathbf{M}$ is introduced in (14). Now, by using (15), we have

$$
\begin{aligned}
\widetilde{v}_{i} & \cong 2^{j} \int_{0}^{1} \mathbf{h}^{T}(x) \mathbf{K} \mathbf{M} \mathbf{P}_{m}^{\alpha} \mathbf{h}(x) h_{i}(x) \mathrm{d} x \\
& =2^{j} \int_{0}^{1} \mathbf{h}^{T}(x) \mathbf{A h}(x) h_{i}(x) \mathrm{d} x,
\end{aligned}
$$


where $\mathbf{A}=\mathbf{K} \mathbf{M} \mathbf{P}_{m}^{\alpha}=\left[a_{i j}\right]_{m \times m}$. It is obvious that $\mathbf{h}^{T}(x) \mathbf{A} \mathbf{h}(x)$ is a $1 \times 1$ matrix, and:

$$
\begin{aligned}
\mathbf{h}^{T}(x) & \mathbf{A} \mathbf{h}(x) h_{i}(x) \\
= & \sum_{m_{1}=2}^{m} \sum_{n_{1}=m_{1}}^{m}\left(a_{n_{1}\left(m_{1}-1\right)}+a_{\left(m_{1}-1\right) n_{1}}\right) \\
& \cdot h_{n_{1}-1}(x) h_{m_{1}-2}(x)+\sum_{i_{1}=1}^{m} a_{i_{1} i_{1}} h_{i_{1}-1}^{2}(x) \\
= & \sum_{n_{1}=2}^{m}\left(a_{1 n_{1}}+a_{n_{1} 1}\right) h_{n_{1}-1}(x) h_{0}(x) \\
& +\sum_{m_{1}=3}^{m} \sum_{n_{1}=m_{1}}^{m}\left(a_{n_{1}}\left(m_{1}-1\right)\right. \\
& \left.+a_{\left(m_{1}-1\right) n_{1}}\right) h_{n_{1}-1}(x) h_{m_{1}-2}(x) \\
& +\sum_{i_{1}=1}^{m} a_{i_{1} i_{1}} h_{i_{1}-1}^{2}(x) \\
= & \sum_{n_{1}=2}^{m}\left(a_{1 n_{1}}+a_{n_{1} 1}\right) h_{n_{1}-1}(x) \\
& +\sum_{m_{1}=3}^{m} \sum_{n_{1}=m_{1}}^{m}\left(a_{n_{1}\left(m_{1}-1\right)}+a_{\left.\left(m_{1}-1\right) n_{1}\right)}\right) \\
& \cdot \rho_{n_{1}-1} m_{1}-2 h_{n_{1}-1}(x) \\
& +\sum_{i_{1}=1}^{m} a_{i_{1} i_{1}} h_{i_{1}-1}^{2}(x) \\
& \\
& \\
& \\
& \\
&
\end{aligned}
$$

where $\rho_{n_{1} m_{1}}$ is defined in (13). Therefore

$$
\begin{aligned}
\widetilde{v}_{i} \cong & 2^{j}\left[\sum_{n_{1}=2}^{m}\left(a_{1 n_{1}}+a_{n_{1} 1}\right) \int_{0}^{1} h_{n_{1}-1}(x) h_{i}(x) \mathrm{d} x\right. \\
& +\sum_{m_{1}=3}^{m} \sum_{n_{1}=m_{1}}^{m}\left(a_{n_{1}\left(m_{1}-1\right)}+a_{\left(m_{1}-1\right) n_{1}}\right) \\
& \cdot \rho_{n_{1}-1 m_{1}-2} \int_{0}^{1} h_{n_{1}-1}(x) h_{i}(x) \mathrm{d} x \\
& \left.+\sum_{i_{1}=1}^{m} a_{i_{1} i_{1}} \int_{0}^{1} h_{i_{1}-1}^{2}(x) h_{i}(x) \mathrm{d} x\right] \\
= & \left(a_{1(i+1)}+a_{(i+1) 1}\right) \\
+ & \sum_{m_{1}=2}^{i}\left(a_{m_{1}(i+1)}+a_{(i+1) m_{1}}\right) \rho_{i\left(m_{1}-1\right)} \\
+ & 2^{j} \sum_{i_{1}=i+2}^{m} a_{i_{1} i_{1}} \rho_{i_{1} i} \frac{1}{2^{l}},
\end{aligned}
$$

where $i_{1}=2^{l}+w$ for $l, w \in\{0\} \cup \mathbb{N}$ and $0 \leq w<2^{l}$. Specifically,

$$
\widetilde{v}_{0}=a_{11}+\sum_{i_{1}=2}^{m} a_{i_{1} i_{1}} \frac{1}{2^{l_{1}}},
$$

for $i_{1}-1=2^{l_{1}}+z$, where $l_{1}, z \in\{0\} \cup \mathbb{N}$ and $0 \leq z<2^{l_{1}}$.

Received: 16 June 2010

Revised: 30 November 2010 